Review

\title{
Social Media Research, Human Behavior, and Sustainable Society
}

\author{
Quan Li ${ }^{1}$, Wenbo Wei ${ }^{1}$, Nian Xiong ${ }^{1}$, Daici Feng ${ }^{1}$, Xinyue Ye ${ }^{2, *}$ and Yongsheng Jiang ${ }^{3, *}$ \\ 1 School of Resource and Environmental Sciences, Wuhan University, Wuhan 430079, China; \\ leequang@whu.edu.cn (Q.L.); wenwb123@163.com (W.W.); xiongnian010@163.com (N.X.); \\ dcfeng028@whu.edu.cn (D.F.) \\ 2 Department of Geography, Kent State University, Kent, OH 44240, USA \\ 3 School of Statistics and Mathematics, Zhongnan University of Economics and Law, Wuhan 430073, China \\ * Correspondence: xye5@kent.edu (X.Y.); jiangys@znufe.edu.cn (Y.J.); \\ Tel.: +1-419-494-7825 (X.Y.); +86-27-8838-5285 (Y.J.); Fax: +1-330-672-4034 (X.Y.); +86-27-8838-5285 (Y.J.)
}

Academic Editor: Paul Burger

Received: 23 January 2017; Accepted: 28 February 2017; Published: 6 March 2017

\begin{abstract}
A bibliometric analysis was conducted to review social media research from different perspectives during the period of 2008-2014 based on the Science Citation Index and Social Science Citation Index database. Using a collection of 10,042 articles related to social media, the bibliometric analysis revealed some interesting patterns and trend of the scientific outputs, major journals, subject categories, spatial distribution, international collaboration, and temporal evolution in keywords usage in social media studies. The research on social media has been characterized by rapid growth and dynamic collaboration, with a rising number of publications and citation. Communication, Sociology, Public, Environment \& Occupational Health, Business, and Multidisciplinary Psychology were the five most common categories. Computers in Human Behavior was the journal with the most social media publications, and Computers \& Education ranked first according to the average citations. The two most productive countries were the U.S. and UK, delivering about half of the publications. The proportion of China's internationally collaborative publications was the highest. The University of Wisconsin, the University of Michigan, and Harvard University were three most productive institutions. Several keywords, such as "Facebook", "Twitter", "communication", "Social Networking Sites", "China", "climate change", "big data" and "social support" increasingly gained the popularity during the study period, indicating the research trends on human behavior and sustainability.
\end{abstract}

Keywords: bibliometric analysis; social media; collaboration; human behavior; sustainable society

\section{Introduction}

Social media platforms are computer-mediated tools that allow people to create, share, or exchange information, career interests, ideas, pictures, and videos in virtual communities and networks. Social media can be defined as "a group of Internet-based applications that build on the ideological and technological foundations of Web 2.0, and that allow the creation and exchange of user-generated content" [1]. Social media is best understood as a group of new kinds of online media that share most or all of the following characteristics: participation, openness, conversation, community, and connectedness. There are basically six kinds of social media including social networks, blogs, wikis, podcasts, forums, content communities, and microblogging [2].

Social media has been evolving and providing world-wide users with information related to the people and events that matter to them. Social media has not only become an indispensable part of our daily lives but also enormously affected global economy and politics. 
Since Mayfield [2] put forward the concept of social media and discussed it systematically, many studies have elucidated the various perspectives of this research field, such as social media users' motivations and personality [3-7], social media usage during disasters [8,9], management on social media sites [10], social media and political engagement [11], social media's impact on business [12-14], predicting elections, depression, flu pandemic [15-17], social media in higher education [18], and social media use among teens and young adults [19].

Although lots of review has been done in the field of social media from the perspective of tourism and hospitality [20], sales process [21], communication [22,23], emergency management [24], higher education [25], and E-government [26], there is still a lack of general overview that can reveal the holistic trend of social media research. Bibliometrics can be used to provide quantitative analysis of literature [27].

Bibliometrics refers to visual and quantitative analytics that are used to summarize trends in selected research fields [28,29]. Bibliometric analyses can reveal temporal dynamics of scholarly outputs, spatial and institutional distributions of publications, scientific collaborations, and major research directions [30-32]. The traditional bibliometric methods usually focus on the citation and content analysis. The emerging bibliometric network analysis often analyzes the relationships among keywords, country, research institute, and author. The common network analysis includes co-word analysis [33-35], co-citation analysis [36,37], co-authorship analysis [38-40], and co-publication analysis [41]. Many disciplines have used bibliometric methods to explore the impact of research themes, such as natural science, engineering, business, social sciences, and humanities [42-46]. However, bibliometric studies have not been performed on the comprehensive social media research literature.

The purpose of this bibliometric study is to analyze the SCI/SSCI scientific literatures on Social Media from 2008 to 2014 by examining the scientific outputs, source titles, science categories, geographical distribution of the authors, international collaborations, and temporal development of keyword frequencies. This study can promote social media research and help researchers in this rapidly growing field.

\section{Data and Methods}

The database was built to use the SCI and SSCI publications on social media. The SCI and SSCI databases are most commonly used for bibliometric studies [47]. We employed the search term "social media". Our bibliographic search resulted in 13,350 social media related publications from the SCI and SSCI databases. Only 10,042 of these papers were reserved after eliminating those without keywords. Then information about each publication was extracted, such as author name and affiliation, subject category, journal name, publication type, publication year, and key words.

The emphasis of the discussion below was to describe global scientific production on social media research from following aspects:

(1) Growth of output during 2008-2014;

(2) Distribution of output in subject categories and journals identified by ISI;

(3) Geographic and institutional distribution of publications;

(4) Distribution of author keywords analysis;

(5) Institution collaboration network analysis.

\section{Results and Discussion}

\subsection{Characteristics of Article Outputs}

Ten thousand and forty-two publications were identified as being social media-related during 2008-2014. The characteristics of article outputs are shown in Figure 1 and Table 1. The annual publications raised from 716 in 2008 to 2509 in 2014, illustrating the dramatic rise and upward 
development of social media research in the past seven years. It is concluded that the annual growth rate of publications obviously accelerated since 2010 through further analysis, and all the annual growth rates except 2008 and 2009 exceeded 20\%. Meanwhile, the average annual growth rate of all SCI and SSCI publications was less than 5\%.

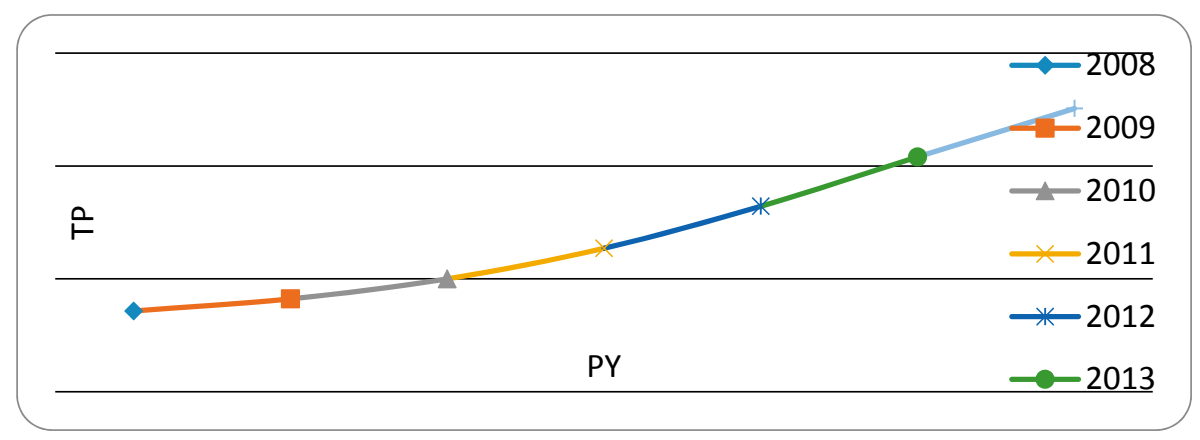

Figure 1. The growth of publication outputs. (PY: year; TP: total of publications).

The average number of authors and references increased from 2.79 and 41.99 in 2008 to 3.03 and 48.62 in 2014, respectively. The growth of references indicated that the accumulation of knowledge about social media has been expanding. However, an interesting fact is the average pages of a paper decreased from 14.97 in 2008 to 14.09 in 2014.

Table 1. Scientific outputs descriptors during 2008-2014.

\begin{tabular}{cccccccccc}
\hline PY & TP & AU & AU/TP & TC & TC/TP & NR & NR/TP & PG & PG/TP \\
\hline 2008 & 716 & 1996 & 2.79 & 8848 & 12.36 & 30,067 & 41.99 & 10,719 & 14.97 \\
2009 & 823 & 2080 & 2.53 & 8580 & 10.43 & 34,692 & 42.15 & 12,001 & 14.58 \\
2010 & 1000 & 2755 & 2.76 & 10,510 & 10.51 & 48,725 & 48.73 & 15,012 & 15.01 \\
2011 & 1271 & 3351 & 2.64 & 8540 & 6.72 & 59,540 & 46.85 & 18,508 & 14.56 \\
2012 & 1643 & 4597 & 2.80 & 7730 & 4.70 & 79,657 & 48.48 & 23,716 & 14.43 \\
2013 & 2080 & 6133 & 2.95 & 4846 & 2.33 & 100,807 & 48.46 & 30,149 & 14.49 \\
2014 & 2509 & 7605 & 3.03 & 1661 & 0.66 & 121,999 & 48.62 & 35,348 & 14.09 \\
total & 10,042 & 28,517 & - & 50,715 & - & 475,487 & - & 145,453 & - \\
average & 1434.6 & 4073.9 & 2.80 & 7245 & 6.80 & $67,926.7$ & 46.50 & 20,779 & 14.60 \\
\hline
\end{tabular}

PY: year; TP: number of publications; AU: number of authors; TC: total citation count; NR: number of cited references; PG: page count; AU/TP, PG/TP, NR/TP, and TC/TP: average of authors, pages, references, and citation in a paper.

\subsection{Subjective Categories and Major Journals}

10,042 social media publications involved 221 ISI-defined subject categories. The top 20 subject categories were presented in Table 2. The five most common categories were Communication (1439 papers; $14.33 \%$ of the total publications), Sociology $(829 ; 8.26 \%)$, Public, Environment \& Occupational Health (735; 7.32\%), Business (627; 6.24\%), and Psychology, Multidisciplinary (575; $5.73 \%)$. Communication was far above any other categories and had the fastest annual growth rate. Articles belonging to top five categories covered $41.88 \%$ of the total articles, while articles from the top 20 categories covered $91.79 \%$. This result illustrated that social media research related to a wider range of disciplines, but its studies were mainly from these 20 categories, especially the top five categories.

These 10,042 social media articles that were published during 2008-2014 appeared in 2360 ISI-indexed journals. The top 10 productive journals were summarized in Table 3, and two of them were indexed by SCI, while nine journals were indexed by SSCI. These 10, or $0.42 \%$ out of the 2360 journals, had published $11.34 \%$ of the total articles. Computers in Human Behavior ranked first and published 236 articles on social media. New Media \& Society published the second most articles (145), followed by Public Relations Review (133), Information Communication \& Society (130), The Journal of Medical Internet Research (107), Media Culture \& Society (92), Comunicar (85), The Journal of Business Ethics 
(83), American Behavioral Scientist (74), and Computers \& Education (55). Social media articles that were published in these journals received, on average, 6.61 citations. Most of these 10 journals belong to communication and sociology. Among these 10 journals, Computers $\mathcal{E}$ Education had average citations of 13.44, and an IF of 2.63, ranked first and second, respectively.

Table 2. Distribution of the subject categories: the top 20.

\begin{tabular}{cc}
\hline SCI/SSCI Subject Category & TP (\%) \\
\hline Communication & $1439(14.33)$ \\
Sociology & $829(8.26)$ \\
Public, Environmental \& Occupational Health & $735(7.32)$ \\
Business & $627(6.24)$ \\
Psychology, Multidisciplinary & $575(5.73)$ \\
Information Science \& Library Science & $563(5.61)$ \\
Education \& Educational Research & $551(5.49)$ \\
Computer Science, Information Systems & $537(5.35)$ \\
Management & $479(4.77)$ \\
Social Sciences, Interdisciplinary & $429(4.27)$ \\
Psychiatry & $335(3.33)$ \\
Psychology, Experimental & $274(2.73)$ \\
Psychology, Clinical & $269(2.68)$ \\
Environmental Studies & $255(2.54)$ \\
Political Science & $233(2.32)$ \\
Linguistics & $231(2.30)$ \\
Environmental Sciences & $222(2.21)$ \\
Geography & $213(2.12)$ \\
Cultural Studies & $211(2.10)$ \\
Health Care Sciences \& Services & $211(2.10)$ \\
Sub Total & $9218(91.79)$ \\
\hline
\end{tabular}

$\mathrm{TP}$, number of publications; \%, the percentage of the subject in the study field .

Table 3. The 10 most active journals in social media research.

\begin{tabular}{ccccc}
\hline Journals & TP (\%) & TC (\%) & TC/TP & IF \\
\hline Computers in Human Behavior & $236(2.35)$ & $1786(3.52)$ & 7.57 & 2.273 \\
New Media \& Society & $145(1.44)$ & $1220(2.41)$ & 8.41 & 2.052 \\
Public Relations Review & $133(1.32)$ & $794(1.57)$ & 5.97 & 0.755 \\
Information Communication E Society & $130(1.29)$ & $629(1.24)$ & 4.84 & 1.283 \\
Journal of Medical Internet Research & $107(1.07)$ & $764(1.51)$ & 7.14 & 4.669 \\
Media Culture E Society & $92(0.92)$ & $361(0.71)$ & 3.92 & 1.139 \\
Comunicar & $85(0.84)$ & $73(0.14)$ & 0.86 & 0.35 \\
Journal of Business Ethics & $83(0.83)$ & $665(1.31)$ & 8.01 & 1.552 \\
American Behavioral Scientist & $74(0.74)$ & $438(0.86)$ & 5.92 & 0.926 \\
Computers E Education & $55(0.55)$ & $739(1.46)$ & 13.44 & 2.63 \\
Total & $1140(11.34)$ & $7469(14.73)$ & & \\
Average & & & 6.61 & 1.76 \\
\hline
\end{tabular}

TP: number of publication; TC: total citation count; TC/TP: average of citations in a paper; IF: ISI Impact factor.

\subsection{Geographic and Institutional Distribution of Publications}

The geographic and institutional distributions of publications were generated based on the affiliation information of authors. We summarized the 10 most productive countries in Figure 2, in terms of the number of total publications, single country articles and international collaborations, respectively. Out of these 10 countries, 7 were from Europe, 1 was from North America, 1 was from Oceania, and 1 was form Asia.

The most productive country was the U.S., owning the most single-country (2810) and international collaborative articles (817). The UK published the second largest number of articles 
(1328). 4955 articles were published by authors from these two countries, accounting for $49.88 \%$ of the total 10,042 articles. The proportion of China's internationally collaborative publications was the highest $(49.79 \%)$. Moreover, it is interesting that Australia was the third most productive. A possible explanation for this is that social media is extraordinarily popular in Australia. According to the Australia Social Media Report 2010, Australia leads the world in average time spent on social media.

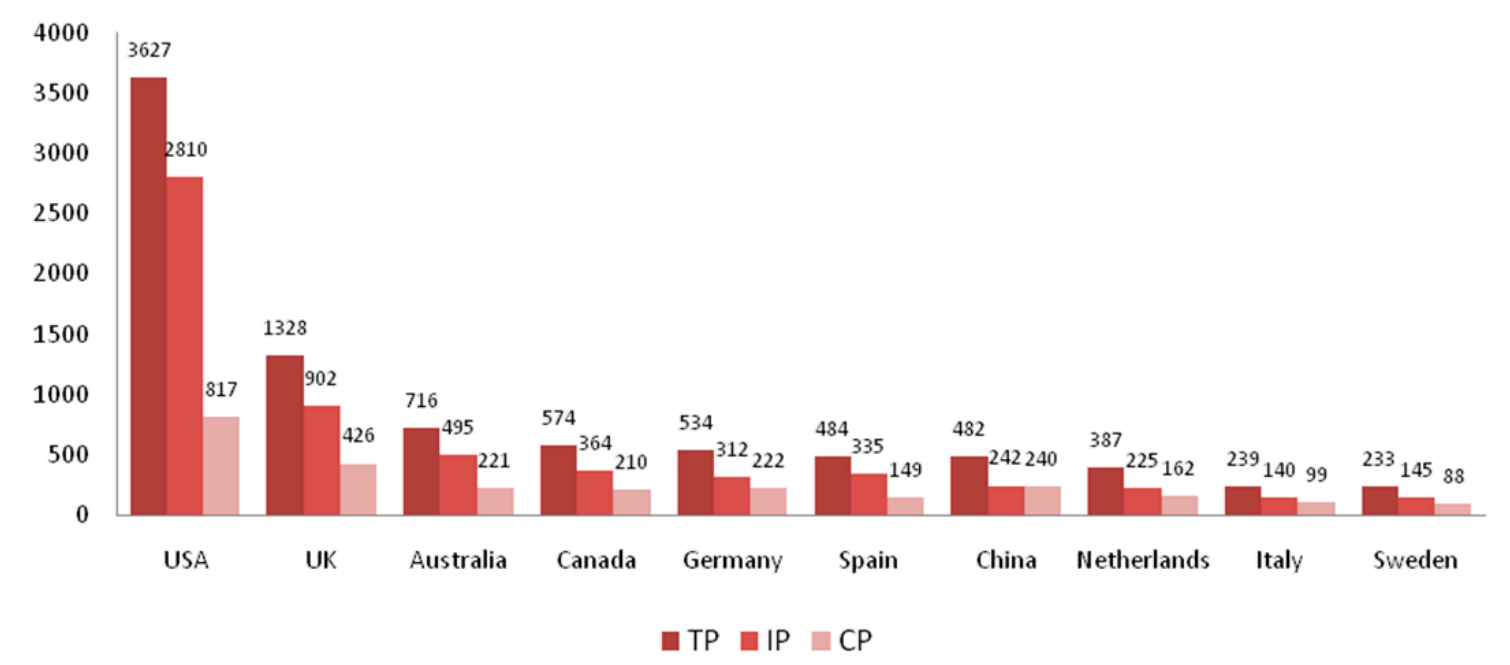

Figure 2. Most productive countries of social media papers during 2008-2014 (TP, total publications; $\mathrm{IP}$, the number of independent publications by single-country; $\mathrm{CP}$, the number of internationally collaborative publications).

\subsection{Institution Collaboration Network}

The Ucinet software calculates the eigenvalue centrality based on the algorithm described as the following [48]: given an adjacency matrix $\mathrm{A}$, the centrality of vertex $i$ (denoted $c_{i}$ ), is given by $c_{i}=a \Sigma \mathrm{A}_{i j} c_{j}$, where $a$ is a parameter. The centrality of each vertex is therefore determined by the centrality of the vertices it is connected to. The parameter $a$ is required to give the equations a non-trivial solution and is therefore the reciprocal of an eigenvalue. It follows that the centralities will be the elements of the corresponding eigenvector. The normalized eigenvector centrality is the scaled eigenvector centrality divided by the maximum difference possible expressed as a percentage.

The collaboration network of the 69 most productive institutes was visualized using the Ucinet software (Figure 3). The node indicates an institute, while the nodal size relates to network eigenvalue centrality in the collaboration network and a nodal color means a specific cluster. As shown in Figure 3, the University Washington and the University Michigan had the highest network eigenvalue centrality. The most productive institution was the University of Wisconsin with 86 papers, followed by the University of Michigan with 82, and Harvard University with 75 . The University of Michigan published the most inter-institutional collaborative publications, followed by Harvard University and the University of Wisconsin. 


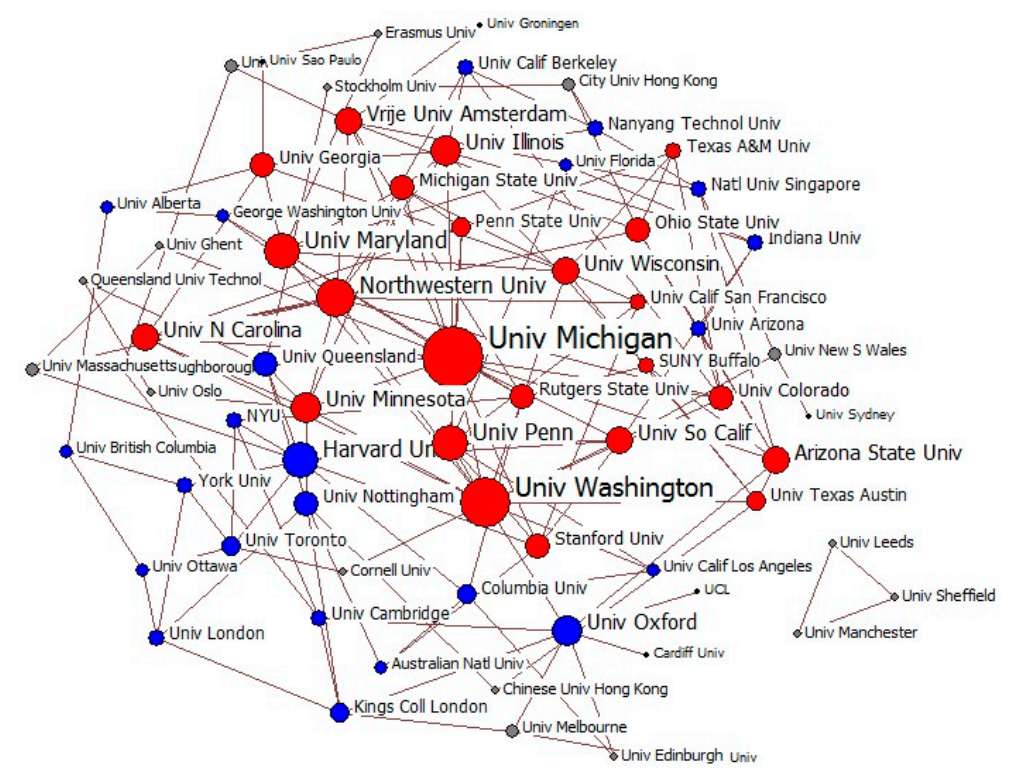

Figure 3. Institution collaboration network of most 69 central institution in social media research (red nodes, $k=4$; blue nodes, $k=3$; grey nodes, $k=2$; black nodes, $k=1$ ).

\subsection{Author Keywords Analysis}

\subsubsection{Temporal Evolution of Author Keywords}

Keywords supplied by the authors offered a profile of article contents. Temporal evolution to these keywords can be used to offer alternative perspective of research hotspots and trends. The 30 most frequently used keywords in the study period were calculated and ranked per year in Table 4.

It is obvious that six keywords ("Facebook", "Twitter", "communication", "Social Networking Sites", "China", and "climate change") received increasing popularity during the study period, likely indicating the hotspots and future research in social media area. The number of papers including author keywords "Facebook" and "Twitter" changed drastically from 0 to 123 and 129 respectively from 2008 to 2014, and these two author keywords ranked 3rd and 2nd respectively in 2014. Moreover, "social networking sites" relating to "Facebook" and "Twitter" also had an obvious upward trajectory in its rank from 179th in 2009 to 8th in 2014. This indicates that the subject of social networking websites itself and special sites such as "Facebook" and "Twitter" received more and more attention during the past five years. Social networking sites, defined by their unique focus on allowing people to "friend" others and share content with other users, are some of the most important kinds of social media. It is estimated that Facebook has 900 million visitors per month, while Twitter has 310 million visitors per month. In addition, "communication" underwent the rank growth from 51st in 2008 to 9th in 2014. This is consistent with the fact that communication with friends and family members is the most common function used on social networking sites, and social media has dramatically changed how we communicate. The analysis above concluded that Communication was the most common subject category for social media. As we expected, "China" increased continuously from 353rd in 2008 to 13th in 2014, suggesting that China remained a hot research topic during the past seven years. This increase concurred with the sharp growth of social media platforms in China, with more than 400-million social media users in 2015. Furthermore, "climate change" has grown from 53rd in 2008 to 24th in 2014, indicating this area has become a new hot spot in social media research. Some keyword such as "big data" and "social support" entered the top 30 keyword list in recent years, to some extent indicating a future research trend in social media area. 
Table 4. Temporal evolution of the 30 most frequently used keywords.

\begin{tabular}{|c|c|c|c|c|c|c|c|c|c|c|c|c|c|c|c|c|c|c|c|c|c|c|c|c|}
\hline \multirow{2}{*}{ DE } & \multicolumn{3}{|c|}{ GROSS } & \multicolumn{3}{|c|}{2008} & \multicolumn{3}{|c|}{2009} & \multicolumn{3}{|c|}{2010} & \multicolumn{3}{|c|}{2011} & \multicolumn{3}{|c|}{2012} & \multicolumn{3}{|c|}{2013} & \multicolumn{3}{|c|}{2014} \\
\hline & $\mathbf{N}$ & $\mathbf{R}$ & $\mathrm{P}(\%)$ & $\mathbf{N}$ & $\mathbf{R}$ & P (\%) & $\mathbf{N}$ & $\mathbf{R}$ & P (\%) & $\mathbf{N}$ & $\mathbf{R}$ & P (\%) & $\mathbf{N}$ & $\mathbf{R}$ & $\mathbf{P}(\%)$ & $\mathbf{N}$ & $\mathbf{R}$ & P (\%) & $\mathbf{N}$ & $\mathbf{R}$ & P (\%) & $\mathbf{N}$ & $\mathbf{R}$ & P (\%) \\
\hline social networking & 427 & 1 & 0.78 & 20 & 3 & $2(0.54)$ & 27 & 3 & $1(0.63)$ & 34 & 3 & $1(0.63)$ & 64 & 3 & $1(0.93)$ & 79 & 2 & $1(0.88)$ & 90 & 4 & $3(0.79)$ & 113 & 4 & $3(0.81)$ \\
\hline Internet & 395 & 2 & 7 & 24 & 2 & $1(0.65)$ & 27 & 2 & $1(0.63)$ & 32 & 4 & $2(0.60)$ & 42 & 4 & $2(0.61)$ & 59 & 4 & $2(0.65)$ & 100 & 2 & $(0.88)$ & 111 & 5 & $4(0.80)$ \\
\hline Facebook $\uparrow$ & 299 & 3 & 0.55 & - & - & 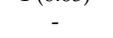 & 5 & 69 & $22(0.12)$ & 4 & 96 & $27(0.07)$ & 20 & 11 & $8(0.29)$ & 56 & 5 & $3(0.62)$ & 91 & 3 & $(0.80)$ & 23 & 3 & $2(0.88)$ \\
\hline Twitter $\uparrow$ & 288 & 4 & & - & - & - & 1 & 773 & $30(0.02)$ & J & 206 & $29(0.06)$ & 19 & 12 & $10(0.28)$ & 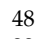 & 6 & $4(0.53)$ & 87 & & $4(0.76)$ & 30 & 2 & $1(0.93)$ \\
\hline Adolesce & 228 & 5 & 2 & 20 & 4 & $2(0.54)$ & 21 & 5 & 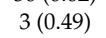 & 26 & 6 & $4(0.49)$ & 31 & 6 & $4(0.45)$ & 33 & 8 & $6(0.37)$ & 38 & 9 & $(0.33)$ & 59 & 7 & $5(0.42)$ \\
\hline Yout & 193 & 6 & 35 & 8 & 17 & $13(0.22)$ & 12 & 15 & 8) & 28 & 5 & $3(0.52)$ & 29 & 8 & $(0.42)$ & 28 & 10 & $8(0.31)$ & 45 & 7 & $5(0.40)$ & 43 & 10 & $8(0.31)$ \\
\hline w & 183 & 7 & 34 & 6 & 28 & $20(0.16)$ & 7 & 30 & $18(0.16)$ & 15 & 11 & $9(0.28)$ & 32 & 5 & $3(0.47)$ & 47 & 7 & $5(0.52)$ & 35 & 12 & $10(0.31)$ & 41 & 11 & $9(0.29)$ \\
\hline $\mathrm{g}$ & 164 & 8 & & 12 & 6 & & 16 & 7 & & & 8 & & & 7 & & & 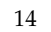 & & & & & & & \\
\hline mass $m$ & 1 & 9 & & 17 & 5 & 4 & 21 & 6 & ) & 22 & 7 & $5(0$ & 2 & 9 & 34) & 28 & 11 & 1) & 28 & & & 22 & 25 & $.16)$ \\
\hline communi & 137 & 10 & - & 4 & 51 & $23(0.11)$ & 12 & 16 & 28) & 9 & 22 & $17(C$ & 15 & 17 & $14(0.22)$ & 16 & 21 & & 36 & 11 & & 45 & 9 & $7(0.32)$ \\
\hline social netwo & 1 & 12 & & & & & 3 & 179 & $29(\mathrm{C}$ & 5 & 79 & & & & & & 15 & & & & & & & \\
\hline 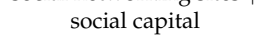 & 122 & 13 & & 12 & 7 & $5(0.33)$ & 12 & 13 & $(0.28)$ & 17 & 9 & $7(0$. & 12 & 27 & $22(0.17)$ & 15 & 28 & 17) & 26 & 15 & & 28 & 20 & .20) \\
\hline social mo & 118 & 14 & 22 & 6 & 29 & $20(0.16)$ & 6 & 37 & $9(0.14)$ & & 24 & $17(0.17)$ & 18 & 14 & $11(0.26)$ & 2 & 13 & & 24 & 18 & & 33 & 15 & $13(0.24)$ \\
\hline & & 15 & & 11 & 9 & & 14 & 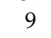 & & 6 & 51 & & 20 & & & & . & & 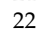 & & & 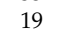 & 30 & 22 \\
\hline & & 16 & & 1 & 353 & & 9 & ? & & 12 & 17 & & 1 & & & & & & & & & & 3 & \\
\hline Content a & 1 & 17 & & 12 & 8 & & 6 & 34 & $19(0$ & 12 & 18 & 1 & 7 & & & 1 & 17 & & 27 & 14 & & 30 & 17 & $15(0.22)$ \\
\hline & & 18 & & 7 & 10 & & 4 & & & & 19 & & 16 & & & & 25 & & 25 & & & 35 & 22 & \\
\hline & 110 & 19 & 0.20 & 11 & 21 & $8(0.30)$ & 15 & 73 & $6(0.35)$ & 11 & 42 & $15(0.21)$ & 18 & 15 & $11(0.26)$ & 15 & 20 & $20(0.17)$ & 16 & 16 & $25(0.14)$ & 24 & 14 & $19(0.17)$ \\
\hline $\begin{array}{l}\text { Corporat } \\
\text { responon }\end{array}$ & 97 & 21 & 0.18 & 7 & 22 & $15(0.19)$ & 14 & 10 & $7(0.32)$ & 12 & 15 & $12(0.22)$ & 14 & 22 & $18(0.20)$ & 15 & 26 & $20(0.17)$ & 19 & 23 & $20(0.17)$ & 16 & 44 & $28(0.11)$ \\
\hline & 99 & 20 & 18 & 10 & 12 & $10(0$ & 12 & 14 & 110 & 14 & 12 & & 14 & 21 & 1 & 16 & 22 & $17(0.18)$ & 16 & 30 & 25 & 17 & 37 & 26 \\
\hline E & 96 & 22 & & 7 & 23 & & 5 & 49 & & 9 & 2 & & 9 & & & & 18 & & 17 & 20 & & 32 & 16 & $14(0.23)$ \\
\hline disco & 84 & 23 & & 9 & 15 & & 13 & 1 & & 16 & 1 & & 11 & & & & & & & & & & & \\
\hline & 80 & 26 & 0 & 10 & 13 & 10 (C & 5 & 48 & $22(0$ & 9 & 25 & 17 & 15 & 18 & 14( & 10 & 51 & 25 & 18 & 26 & 22 & 13 & 53 & $29(0.09)$ \\
\hline & 80 & 27 & & 1 & 354 & & 10 & 19 & & 14 & 13 & & 15 & & & & & & & & & & & \\
\hline Clin & 79 & 2 & & 4 & 53 & & 5 & 5 & & . & 14 & & 10 & & & 1 & & & 19 & & & 23 & & \\
\hline & 78 & 2 & & 7 & 24 & & 5 & 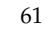 & & 11 & 2 & & 9 & 4 & & 9 & 75 & & 18 & 7 & & 19 & 32 & $22(0.14)$ \\
\hline Trust & 75 & 30 & 0.14 & 7 & 22 & $15(0.19)$ & 4 & 75 & $27(0.09)$ & 7 & 45 & $23(0.13)$ & 10 & 35 & $25(0.15)$ & 10 & 51 & $25(0.11)$ & 11 & 62 & $30(0.10)$ & 26 & 21 & $18(0.19)$ \\
\hline
\end{tabular}

$\mathrm{DE}$, author keywords; $\mathrm{N}$, articles in the study period; $\mathrm{R}$, the absolute rank of author keywords; $\mathrm{P}$, the relative rank of author keywords; \%, the percentage of author keywords ; -, no such author keyword in specific time period; $\uparrow$, rising trend; $\downarrow$, declining trend. 
The ranks of some of the most frequently used keywords during 2008-2014 retained relative stabilization. This indicates that these keywords are hot issues in social media study. "Adolescents", "youth" and "children" means young people is hot issue in social media research during past 7 years. "Web 2.0", the technology base of social media, is always an important subject during 2008-2014. "social capital", "social movement", "education", "ethics", "public health", "culture", and "journalism", which concerned sociology, education, health, and culture and media fields, suggested that social media research connected tightly with the application fields and had the characteristics of interdisciplinary and multi perspective.

On the contrary, the rank of "discourse" experienced an apparent decline during the study period, and the ranks of "mass media", "gender", and "risk" have also dropped in recent years. In addition, "obesity", "HIV/AIDS", "body image", "violence", and "qualitative research" that used to be 30 most frequently adopted keywords have faded away in most recent years.

\subsubsection{Author Keywords Co-Work Network Analysis}

Co-occurrence relationships among top 90 high-frequency keywords were examined, and the co-word networks were visualized (Figure 4). The nodes are high-frequency author keywords, the size of which represents the value of network eigenvalue centrality. The higher value means the stronger the connectivity and control. The segment indicates the connection relationship between two words. Moreover, the different colors indicate degree of core or edge, and the red nodes stand for the core themes.

As shown in Figure 4, the author keywords with the highest network eigenvalue centrality were "social networking" and "Internet", and the other core themes including social networking sites correlative themes such as "Facebook", "Twitter", "YouTube", and "social networking sites"; technology correlative themes such as "Web 2.0", "technology", and "content analysis"; concept concerned themes such as "new media", "digital media", and "mass media"; industry concerned themes such as "education" and "journalism"; and interested points concerned themes such as "climate change", "globalization", "politics", "community", "culture", "democracy", "youth", "adolescents", "ethnography", "race", "gender", "identity", and "privacy". This is all evidence for the research hotspots in social media field during the study period.

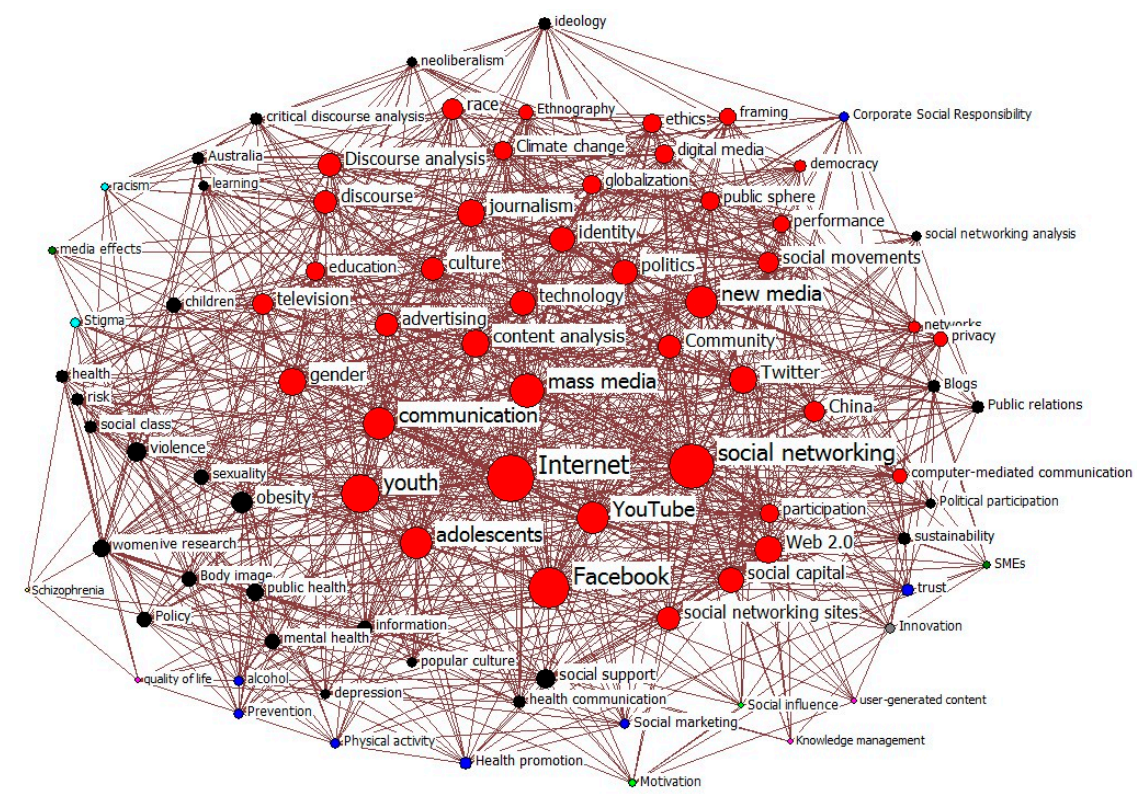

Figure 4. Co-work network of top 90 high-frequency author keywords (co-work network, red nodes, $k=18$; blue nodes, $k=16$; black nodes, $k=15$; grey nodes, cyan nodes, $k=13$; $k=12$; dark green node, $k=11$; light green node, $k=10$; pink nodes, $k=8$; yellow nodes, $k=6$ ). 


\section{Conclusions}

From 2008 to 2014, the annual publications of social media increased from 716 to 2509, with an average annual growth rate of more than $20 \%$. A total of 10,042 articles were listed in 221 ISI-defined subject categories and appeared in 2360 ISI-indexed journals. The five most common categories in the publications were Communication, Sociology, Public, Environment \& Occupational Health, Business and Multidisciplinary Psychology, accounting for $41.88 \%$ of all articles. In addition, the five most productive journals on social media were Computers in Human Behavior, New Media \& Society, Public Relations Review, Information Communication \& Society, and The Journal of Medical Internet Research. Among these journals, Computers $\mathcal{E}$ Education had the average citations of 13.44 and an IF of 2.63 , ranked 1st and 2nd respectively.

The geographic and institutional distributions of publications suggested that the most productive country was the U.S., contributing the most single-country and international collaborative articles. UK published the second highest number of articles. These two countries published 4955 articles, $49.88 \%$ of the total articles. Out of these top 10 productive countries, 7 were from Europe, 1 was from North America, 1 was from Oceania, and 1 was from Asia.

A keywords analysis through temporal evaluation and co-work network analysis social networking sites demonstrated that technology, concept, industry, and interested points were consistent topics that grabbed the most attention during the study period. Several keywords, such as "Facebook", "Twitter", "communication", "Social Networking Sites", "China", "climate change", "big data", and "social support" attracted increasing attention, indicating future research trends.

The collaboration network of the top 69 most productive institutes suggested the University of Washington and the University of Michigan owned the highest network eigenvalue centrality. The most productive institution was the University of Wisconsin, followed by the University of Michigan and Harvard University. The University of Michigan published the most inter-institutional collaborative publications, followed by Harvard University and the University of Wisconsin. The analytical framework suggested in this paper can be expanded to databases other than SCI/SSCI and other time periods.

Acknowledgments: This material is partially based upon work supported by the National Science Foundation under Grant Nos. 1416509 and 1637242. Any opinions, findings, and conclusions or recommendations expressed in this material are those of the author and do not necessarily reflect the views of the National Science Foundation.

Author Contributions: Quan Li, Wenbo Wei, and Xinyue Ye conceived and designed the study and outlined the methodology; Quan Li and Wenbo Wei analyzed the data; Nian Xiong and Daici Feng contributed materials; Yongsheng Jiang extensively updated the manuscript. All authors have read and approved the final manuscript.

Conflicts of Interest: The authors declare no conflict of interest.

\section{References}

1. Kaplan, A.M.; Haenlein, M. Users of the world, unite! The challenges and opportunities of social media. Bus. Horiz. 2010, 1, 59-68. [CrossRef]

2. Mayfield, A. What Is Social Media? 2008. Available online: http://www.repromax.com/docs/113/ 854427515.pdf (accessed on 5 April 2015).

3. Ryan, T.; Xenos, S. Who uses Facebook? An investigation into the relationship between the Big Five, shyness, narcissism, loneliness, and Facebook usage. Comput. Hum. Behav. 2011, 5, 1658-1664. [CrossRef]

4. Smock, A.D.; Ellison, N.B.; Lampe, C.; Donghee Yvette, W. Facebook as a toolkit: A uses and gratification approach to unbundling feature use. Comput. Hum. Behav. 2011, 6, 2322-2329. [CrossRef]

5. Cheung, C.M.K.; Chiu, P.; Lee, M.K.O. Online social networks: Why do students use facebook? Comput. Hum. Behav. 2011, 4, 1337-1343. [CrossRef]

6. Kima, Y.; Sohn, D.; Choi, S.M. Cultural difference in motivations for using social network sites: A comparative study of American and Korean college students. Comput. Hum. Behav. 2011, 1, 365-372. [CrossRef]

7. Hughes, D.J.; Rowea, M.; Batey, M.; Lee, A. A tale of two sites: Twitter vs. Facebook and the personality predictors of social media usage. Comput. Hum. Behav. 2012, 2, 561-569. [CrossRef] 
8. Wang, Z.; Ye, X.; Tsou, M. Spatial, temporal, and content analysis of Twitter for wildfire hazards. Natl. Hazards 2016. [CrossRef]

9. Kaigo, M. Social Media Usage during Disasters and Social Capital: Twitter and the Great East Japan Earthquake. Keio Commun. Rev. 2012, 34, 19-35.

10. Leskovec, J.; Huttenlocher, D.; Kleinberg, J. Governance in Social Media: A Case Study of the Wikipedia Promotion Process. In Proceedings of the 4th International AAAI Conference on Weblogs and Social Media, Washington, DC, USA, 23-26 May 2010; pp. 98-105.

11. Rainie, L.; Smith, A.; Schlozman, K.L.; Brady, H.; Verba, S. Social Media and Political Engagement. Available online: http:/ / pewinternet.org/Reports/2012/Political-Engagement.aspx (accessed on 23 January 2017).

12. Smith, T. The social media revolution. Int. J. Mark. Res. 2009, 4, 559-561. [CrossRef]

13. Miller, R.; Lammas, N. Social media and its implications for viral marketing. Asia Pac. Public Relat. J. 2010, $11,1-9$.

14. Edosomwan, S.; Prakasan, S.K.; Kouame, D.; Watson, J.; Seymour, T. The History of Social Media and its Impact on Business. J. Appl. Manag. Entrep. 2011, 16, 3.

15. Ritterman, J.; Osborne, M.; Klein, E. Using prediction markets and Twitter to predict a swine flu pandemic. In Proceedings of the 1st International Workshop of Mining Social Media, Sevilla, Spain, 9 November 2009; pp. 9-17.

16. Tumasjan, A.; Sprenger, T.O.; Sandner, P.G.; Welpe, I.M. Predicting Elections with Twitter: What 140 Characters Reveal about Political Sentiment. In Proceedings of the 4th International AAAI Conference on Weblogs and Social Media, Washington, DC, USA, 23-26 May 2010.

17. Ye, X.; Li, S.; Yang, X.; Qin, C. Use of Social Media for Detection and Analysis of Infectious Disease in China. ISPRS Int. J. Geo-Inf. 2016. [CrossRef]

18. Selwyn, N. Social Media in Higher Education; The Europa World of Learning: Lodon, UK, 2012. Available online: https://www.researchgate.net/publication/265159738_SOCIAL_MEDIA_IN_HIGHER_ EDUCATION (accessed on 1 March 2017).

19. Lenhart, A.; Purcell, K.; Smith, A.; Zickuhr, K. Social Media and Young Adults. Available online: http: //www.pewinternet.org/Reports/2010/Social-Media-and-Young-Adults.aspx (accessed on 23 January 2017).

20. Leung, D.; Law, R.; van Hoof, H.; Buhalis, D. Social Media in Tourism and Hospitality: A Literature Review. J. Travel Tour. Mark. 2013, 1-2,3-22. [CrossRef]

21. Andzulis, J.; Panagopoulos, N.G.; Rapp, A. A Review of Social Media and Implications for the Sales Process. J. Pers. Sell. Sales Manag. 2013, 3, 305-316. [CrossRef]

22. Moorhead, S.A.; Hazlett, D.E.; Harrison, L.; Carroll, J.K.; Irwin, A.; Hoving, C. A New Dimension of Health Care: Systematic Review of the Uses, Benefits, and Limitations of Social Media for Health Communication. J. Med. Internet Res. 2013, 4, 85. [CrossRef] [PubMed]

23. Veil, S.R.; Buehner, T.; Palenchar, M.J. Work-In-Process Literature Review: Incorporating Social Media in Risk and Crisis Communication. J. Conting. Crisis Manag. 2011, 2, 110-122. [CrossRef]

24. Crowe, A. The social media manifesto: A comprehensive review of the impact of social media on emergency management. J. Bus. Contin. Emerg. Plan. 2010, 1, 409-420.

25. Tess, P.A. The role of social media in higher education classes (real and virtual)—A literature review. Comput. Hum. Behav. 2013, 5, 60-68. [CrossRef]

26. Magro, M.J. A Review of Social Media Use in E-Government. Adm. Sci. 2012, 2, 148-161. [CrossRef]

27. De Bellis, N. Bibliometrics and Citation Analysis: From the Science Citation Index to Cybermetrics; Scarecrow Press: Lanham, MD, USA, 2009; Volume 417.

28. Pritchard, A. Statistical bibliography or bibliometrics? J. Doc. 1969, 25, 348-349.

29. Garfield, E. Citation indexing for studying science. Nature 1970, 227, 669-671. [CrossRef] [PubMed]

30. Chiu, W.T.; Huang, J.S.; Ho, Y.S. Bibliometric analysis of severe acute respiratory syndrome-related research in the beginning stage. Scientometrics 2004, 61, 69-77. [CrossRef]

31. Li, J.; Zhang, Y.; Wang, X.; Ho, Y.S. Bibliometric analysis of atmospheric simulation trends in meteorology and atmospheric science journals. Croat. Chem. Acta 2009, 82, 695-705.

32. Zhang, W.; Qian, W.; Ho, Y.S. A bibliometric analysis of research related to ocean circulation. Scientometrics 2009, 80, 305-316. [CrossRef]

33. Zhao, L.; Zhang, Q. Mapping knowledge domains of Chinese digital library research output, 1994-2010. Scientometrics 2011, 89, 51-87. [CrossRef] 
34. Ding, Y.; Chowdhury, G.; Foo, S. Bibliometric cartography of information retrieval researchby using co-word analysis. Inf. Process. Manag. 2011, 37, 817-842. [CrossRef]

35. Niu, B.; Hong, S.; Yuan, J.; Peng, S.; Wang, Z.; Zhang, X. Global trends in sediment-related research in earth science during 1992-2011: A bibliometric analysis. Scientometrics 2014, 98, 511-529. [CrossRef]

36. He, Y.; Hui, S. Mining a web citation database for author co-citation analysis. Inf. Process. Manag. 2002, 38, 491-508. [CrossRef]

37. Lai, K.; Wu, S. Using the patent co-citation approach to establish a new patent classification system. Inf. Process. Manag. 2005, 41, 313-330. [CrossRef]

38. Glanzel, W. Science in Scandinavia: A bibliometric approach. Scientometrics 2000, 48, 121-150. [CrossRef]

39. Seglen, P.; Aksnes, D. Scientific productivity and group size: A bibliometric analysis of Norwegian microbiological research. Scientometrics 2000, 49, 125-143. [CrossRef]

40. Liu, X.; Zhan, F.; Hong, S.; Niu, B.; Liu, Y. A bibliometric study of earthquake research: 1900-2010. Scientometrics 2012, 92, 747-765. [CrossRef]

41. Schmoch, U.; Schubert, T. Are international co-publications an indicator for quality of scientific research? Scientometrics 2008, 74, 361-377. [CrossRef]

42. De Bakker, F.G.A.; Groenewegen, P.; Hond, F.D. A Bibliometric Analysis of 30 Years of Research and Theory on Corporate Social Responsibility and Corporate Social Performance. Bus. Soc. 2005, 3, 283-317. [CrossRef]

43. Nederhof, A.J. Bibliometric monitoring of research performance in the Social Sciences and the Humanities: A Review. Scientometrics 2006, 1, 81-100. [CrossRef]

44. Tarkowski, S.M. Environmental health research in Europe-Bibliometric analysis. Eur. J. Public Health 2007, 17, 14-18. [CrossRef] [PubMed]

45. Li, T.; Ho, Y.S.; Li, C.Y. Bibliometric analysis on global Parkinson's disease research trends during 1991-2006. Neurosci. Lett. 2008, 441, 248-252. [CrossRef] [PubMed]

46. Xie, S.; Zhang, J.; Ho, Y.S. Assessment of world aerosol research trends by bibliometric analysis. Scientometrics 2008, 77, 113-130. [CrossRef]

47. Liu, X.; Zhang, L.; Hong, S. Global biodiversity research during 1900-2009: A bibliometric analysis. Biodivers. Conserv. 2010, 20, 807-826. [CrossRef]

48. DeJordy, R.; Halgin, D.; Everett, M.; Borgatti, S. Social Network Analysis Software. Available online: http:/ / www.analytictech.com (accessed on 1 March 2017).

(C) 2017 by the authors. Licensee MDPI, Basel, Switzerland. This article is an open access article distributed under the terms and conditions of the Creative Commons Attribution (CC BY) license (http://creativecommons.org/licenses/by/4.0/). 